\title{
SECCIÓN ARQUEOLÓGICA
}

\author{
$F U L \bar{U} S$ Y MONEDA EN MALLORCA, IBIZA \\ Y MENORCA ANTES DEL 290 H/902 D. C.
}

Fèlix Retamero

Universidad Autónoma de Barcelona

\begin{abstract}
Afortunadamente, el registro numismático conocido de piezas de cobre del siglo I H./vII-VIII d. C. va siendo, aunque en proporciones muy modestas, paulatinamente ampliado. Posiblemente, el escaso interés de los coleccionistas por estos ejemplares revierta en la discreción con que estas piezas aparecen en las contribuciones numismáticas. En cualquier caso, cuando lo hacen, acostumbran a formar parte de noticias sobre conjuntos de monedas con cronologías extensas, hallados en una zona o depositados en alguna institución ${ }^{1}$. Es decir, los fulūs -y no exclusivamente los de los siglos I y II H.- no parecen tener suficiente entidad numismática como para merecer una atención específica ${ }^{2}$.

Seguramente, la causa de esta pobre atención, que en el mejor de los casos proporciona valiosos catálogos y cronologías afinadas, haya que buscarla, al menos en parte, en una inercia intelectual consistente en presuponer, para cada tipo metálico, diferentes funciones monetarias. Así, se atribuye a la moneda de cobre, de manera corriente, un poder adquisitivo bajo; se la considera como el instrumento adecuado \footnotetext{
numerario árabe en la comarca de Monzón", Rubartayr, 0 ( = Gaceta Numismática, 105 106, junio-septiembre, 1992, pp. 165-178); Domènech, C.; Trelis, J., «Hallazgos numismáticos de época islámica en Crevillente (Alicante)", III Jarique de Numismática Hispano-Arabe, Madrid, 1992, pp. 333-346; Gomis, M., "El monetario del Museo de Nules: contribución al estudio de la circulación monetaria en la zona», Gaceta Numismática, 111, diciembre (1993), pp. 55-66.

2 Afortunadamente, éste no es el caso de las emisiones de oro del mismo período, sobre las que existe, comparativamente, una extensa bibliografía. Véase, entre otros trabajos, Navascués, J., "Los sueldos hispano-árabes", Numario Hispánico, 8 (1959), pp. 15-33; Balaguer, A. M., Las emisiones transicionales árabe-musulmanas en Hispania, 1976; Balaguer, A. M.; Bates, M., «Early Islamic Gold Issues of North Africa and Spain in the American Numismatic Society", Museum Notes, 24 (1979), pp. 225-241; Bates, M., "The Coinage of Spain Under the Umayyad Caliphs of the East, 711-750", III Jarique de Numismática Hispano-Arabe, Madrid, 1992, pp. 271-290.
}

1 Algunos ejemplos recientes pueden encontrarse en López, P., "La circulación del
\end{abstract}


para las pequeñas transacciones cotidianas $\mathrm{y}$, finalmente, como una moneda usada en espacios restringidos ${ }^{3}$.

Aparte de presentar los fulüs encontrados en Mallorca, Menorca e Ibiza, incluidos en el catálogo adjunto, lo que se pretende en este artículo es cuestionar, no la veracidad de los usos atribuidos a este tipo de piezas, sino el alcance explicativo de tales supuestos. Por otro lado, el hecho de que estos fulüs hayan aparecido en estas islas (hay constancia de hallazgos monetarios en Formentera, pero, de momento, no ha sido posible acceder a estas piezas) permite añadir, a la cuestión de la introducción y acuñación de estas monedas en al-Andalus, un problema adicional: la presencia, en las islas, de piezas acuñadas casi dos siglos antes de la expedición comandada por 'Ișām al-Jawlānī en el 902 d. C.

Sobre el primer punto, a la falta de una carta exhaustiva de hallazgos ${ }^{4} \mathrm{y}$ al desconocimiento de la magnitud de ejemplares que circularon en al-Andalus, convendría añadir el problema de la prolijidad tipológica y el de la relación de estas series acuñadas sobre metal «vil» con las emisiones de piezas de oro.

Los seis ejemplares -dos por isla- que se presentan en el catálogo proceden de colecciones particulares. Los encontrados en Ibiza fueron legados póstumamente al museo de esta isla y formaban parte de un conjunto más amplio ${ }^{5}$.

Si bien se trata de piezas aisladas halladas superficialmente, los lugares de procedencia, en cada una de las tres islas, son los mismos: Sa Coma, en Ibiza; La Mola de Felanitx, en Mallorca y Tot Lluc, en Menorca. Es decir que, de momento, la evidencia disponible muestra una concentración de los hallazgos coincidente en las tres islas.

Dejando de lado la pieza frustra hallada en Sa Coma (Ibiza) y el fals con la inscripción nafaqa fi sabil Allāh en el anverso de La Mola de Fe-

\footnotetext{
3 Así, recientemente se ha propuesto que las emisiones de cobres visigodos sirvieron para cubrir las necesidades derivadas del pequeño comercio ciudadano. Crusafont M., El sistema monetario visigodo: cobre y oro, ANE. Museo Casa de la Moneda, Barcelona-Madrid, 1994.

4 Corpus de hallazgos monetarios andalusies. Estudio (M. Barceló, A. Canto). Corpus (A. M. Balaguer, A. Canto). En prensa.

5 Retamero, F., Moneda i monedes àrabs a l'illa d'Eivissa. Treballs del Museu Arqueològic d'Eivissa i Formentera, Ibiza, 1995.
} 
lanitx (Mallorca) ${ }^{6}$, los cuatro ejemplares restantes contienen las mismas leyendas: محمـد ر/سول الله en el anverso y el اله //الا الله en el reverso.

Sobre la procedencia y las fechas de emisión de los fulūs con la inscripción نفقـــ poco se puede añadir a las sugerencias de J. Walker. Aunque con reservas, Walker proponía la ceca de Tanŷa como centro emisor y situaba estas acuñaciones, perhaps on the eve of their successful raid accross the straits of Gibraltar in A.H. 92, es decir, en una fecha inmediatamente anterior al $711^{7}$. Difícilmente puede sostenerse la traducción de nafaqa fi sabil Alläh como the pay given to warriors, who went forth to fight 'in the Way of Allah'y, en consecuencia, deducir, únicamente del estricto contenido de la leyenda, una relación entre estas emisiones y el pago de las soldadas requeridas para la conquista de Tanŷa o de Hispania 8 . Sin embargo, la evidencia numismática proporcionada por los ejemplares con la leyenda nifäq tayyib 'an Allāh y acuñados en Țanŷa permite, de momento, y por extensión, pensar que la pieza con la inscripción nafaqa encontrada en La Mola de Felanitx procede de esta ceca ${ }^{9}$.

Las leyendas de los otros ejemplares (núms. 2, 3, 5 y 6) tampoco permiten precisar el lugar y la fecha de emisión. Existen, no obstante, piezas catalogadas con estas inscripciones y con la misma distribución de las leyendas centrales acuñadas en al-Andalus en el 108 H/726-7 d. C. ${ }^{10}$, si bien la combinación de la profesión de fe y la misión profética de Muhammad se encuentra ya en piezas bilingües de oro, acuñadas

${ }^{6}$ Esta pieza ha sido recientemente publicada por Barceló, M., «Els fulūs de Țanğa de finals del segle I h/vir d. C., els pactes més antics i el cas de Mallorca i de Menorca: una revisió", Gaceta Numismática, 114, noviembre (1994), pp. 5-18, y "Correccions", Gaceta Numismática, 115, p. 63-64.

7 Walker, J., A Catalogue of the Arab-Byzantine and Post-Reform Umaiyad Coins, London (en adelante, Walker), pp. LXVIII-LXIX.

8 Walker, p. LXVIII; Barceló, M., "Un fals de yihäd encunyat a Țanŷa probablement abans de 92/711", Acta Numismática, VII (1977), pp. 187-189. Para una revisión del significado de las inscripciones nafaqa y nifä $q$ en estas piezas, véase Barceló, M., "Els fulüs de Tanǧa...", pp. 6-8.

9 Tiesenhausen, W., Moneti vostochnavo Khalifata, St. Petersburg, 1873, núm. 2609, p. 26; Nützel, H., Katalog der orientalischen Münzen, Berlín, 1902 (en adelante, Nützel), núm. 2025 = Walker, p. 271, B52; Barceló, M., "Un fals de ŷihäd...", p. 189.

10 Gaillard, J., Description des monnaies espagnoles et des monnaies étrangères jusqu'à nos jours, Madrid, 1852, p. 346, núms. 5838-5740; Miles, G. C., The Coinage of the Umayyads of Spain, I, New York, 1950, núm. 8 (b), p. 119. Walker, p. 233, núm. 759. En nota a pie de página, Walker indicaba que In fact the earliest certain date on the copper coins of al-Andalus is 108. 
en el Norte de Africa desde el año 97 H/715-6 d. C. ${ }^{11}$, en al-Andalus desde el $98 \mathrm{H}$ y en fulūs de Ifrīqiya acuñados entre el $97-99 \mathrm{H}{ }^{12}$. Posiblemente, un estudio de los contenidos metálicos de estas piezas, contrastados con los de aquellos ejemplares de procedencia y fechas conocidas, ayudaría a establecer unos orígenes y cronología más afinados ${ }^{13}$.

Mientras tanto, sin embargo, el hecho de que piezas acuñadas -bien en el Norte de Africa, bien en al-Andalus- en los últimos años del siglo i $\mathrm{H}$ y a comienzos del II $\mathrm{H}$ circularon en las Islas Baleares parece indiscutible. Esta constatación, por otro lado, da pie a plantear diversos problemas. La primera cuestión notable es la magnitud de la presencia de estos fulūs, no sólo en las islas, sino también en al-Andalus. Cualquier estimación en este sentido, a falta de un inventario de piezas, de estudios de cuños ${ }^{14} \mathrm{y}$ de un mapa exhaustivo y de actualización continua de los hallazgos, sería, de momento, precipitada. Este panorama, junto a la pequeñez de la muestra manejada, hacen vano cualquier intento de resolución. El único ejercicio posible, por ahora, consiste en sopesar estos hallazgos tomando como referencia los registros de piezas árabes perdidas, todavía inéditos, de dos de estas islas, Ibiza y Menorca ${ }^{15}$.

Los hallazgos de ejemplares procedentes de pérdidas involuntarias han sido utilizados recientemente como indicadores de los volúmenes

11 Walker, pp. 78-79; Balanguer, A. M., Las emisiones transicionales..., p. 70, y núms. 53 y 54 . El inicio de las emisiones bilingües andalusíes supuso, según $M$. Bates, la definitiva separación de las cecas de al-Andalus y del Norte de Africa. Por otro lado, Bates sugiere la posibilidad de que el inicio de las emisiones de fulūs andalusíes con estrella en el anverso coincidiera con las acuñaciones de solidi bilingües. Bates, M., "The coinage of Spain...", p. 284.

12 Walker, pp. 231, P123.

13 Manteniendo, sin embargo, ciertas reservas. Un estudio de piezas de cobre vándalas, por ejemplo, ha revelado que los porcentajes de los diferentes metales que forman la aleación pueden variar tanto según los talleres como entre diferentes emisiones de una misma ceca. King, C. E., et al., "Copper-based Alloys of the Fifth Century. A Comparison of Carthage under Vandalic Rule, with other Mints", Revue Numismatique, XXXIV (1992), pp. 54-76.

14 Que, por otro lado, deberán abordar el problema de la vida productiva de los cuños utilizados, marcada tanto por los constreñimientos técnicos como por eventuales decisiones tomadas por las autoridades de la ceca.

15 Retamero, F., Moneda $i$ monedes àrabs... pp. 17-18. Una parte, sin embargo, fue publicada en Rosselló, G., Notas para un estudio de Ibiza musulmana, Eivissa, 1985. El segundo será publicado por Bernat Moll i Mercadal, "Contribució a l'estudi de la circulació monetària a la Menorca musulmana", Meloussa, 3 (1994) pp. 25-68. Agradezco a su autor la amabilidad de haberme facilitado el borrador de este importante corpus de hallazgos. 
relativos de piezas en circulación. Entre otras ventajas de este procedimiento estimativo, se ha señalado el hecho de que los ejemplares perdidos accidentalmente permiten evitar la tendencia de los depósitos monetarios a contener aquellas piezas consideradas "mejores" según la "Ley" de Gresham. De aquí que estos hallazgos son more representative of the currency, although perhaps favouring the smaller denominations that are more easily lost and less easily found ${ }^{16}$.

Una primera comparación de los registros numismáticos de Ibiza y Menorca permite avanzar que, en términos relativos, y de manera coincidente en ambos casos, el primer momento de presencia voluminosa de piezas de moneda -en relación, por supuesto, al tamaño de las muestras- corresponde al período taifa (siglo v H/XI d. C.). Esta estimación en términos comparativos deberá tener en cuenta, de todas maneras, la distribución de los hallazgos para determinar si, en el fondo de este incremento, se aprecia una extensión territorial de la presencia monetaria, o bien si ésta se circunscribe, de manera intensa, a zonas limitadas.

Provisionalmente, pues, parece razonable proponer que la introducción de fulūs de los siglos I-II H/vII-VIII d. C. no se produjo de manera voluminosa ni extensa, teniendo en cuenta el número absoluto de piezas, los valores relativos respecto a otros períodos y la reiteración, hasta el momento, de los escasos hallazgos en los mismos lugares.

El segundo problema que hay que tener en cuenta es el del momento y ritmos de la introducción de estos fulüs en las islas. Una persistente repetición de cuños en piezas del mismo tipo podría sugerir que la penetración se hizo de una manera compacta, de una sola vez. Aunque las cinco piezas catalogables tan sólo corresponden a dos tipos diferentes y uno de ellos está representado por un único ejemplar, un primer $-\mathrm{y}$

16 Blackburn, M. A. S., "Single-finds as a measure of monetary activity in the Early Middle Ages", Seria Numismatyczna i Konserwatorska, 9 (1989), pp. 67-85. El principio general que rige este procedimiento es bien sencillo: la probabilidad de que una pieza sea perdida es proporcional a su número. Müller, J. W., "Quelques remarques sur le poids originel des monnaies usées", Revue Numismatique, XIX (1977), pp. 190-198, p. 191. Sobre las diferencias de representatividad de las llamadas piezas de "alto" y "bajo" valor, véase Williams, H., "Coin Supply in Britain in the Late Third Century as Evidenced by a Mathematical Interpretation of Site Finds", Numismatic Chronicle, 152 (1992) pp. 49-56, p. 49. Para una crítica de la "Ley" de Gresham, véase Hennequin, G., "Bonne' ou 'mauvaise' monnaie? Mutations monétaires et loi de Gresham avant l'époque moderne", L'Information Historique, XXXIX (1977), pp. 203-212, y Rolick, A. J., y Weber, W. E., "Gresham's Law or Gresham's Fallacy", Journal of Political Economy, 94-1 (1986), pp. 185-199. 
casi ridículo- estudio de los cuños revela una diversidad total: las piezas con la profesión de fe en el anverso y la misión profética de Muḥammad en el reverso recogidas en el catálogo (Lavoix, $1333{ }^{17}$; Miles, 26; Walker, 681-684) fueron estampadas con cuños, tanto de anverso como de reverso, diferentes.

Con la intención de ampliar la muestra estudiada, se ha incluido un conjunto de seis piezas del mismo tipo, conservadas en el Museu de Menorca. En principio, no hay pruebas concluyentes de que estos ejemplares, que forman parte de un lote de monedas árabes de cecas y períodos diversos, fueran hallados en Menorca o en alguna de las otras islas. Se piensa que este grupo de piezas formó parte de la colección Vives Escudero. Hay razones para dudar, sin embargo, de esta adscripción y para sugerir el origen isleño de todo este conjunto. En primer lugar, B. Moll ha señalado recientemente la existencia, en este lote, de ejemplares de época taifa inéditos, inexplicablemente ausentes en los catálogos del propio Vives y de Prieto y Vives ${ }^{18}$. Por otro lado, tal como sucede en los conjuntos de hallazgos de Ibiza y Menorca, la parte más importante de este lote de 149 piezas ${ }^{19}$ (aproximadamente, tres cuartas partes) está formada por piezas de época taifa, entre las que destacan, por su número, las acuñadas en Madinat Mayūrqa por alMurtaḍà y Mubaššir (54 ejemplares) y las emitidas a nombre de 'Alī Iqbāl al-Dawla (21 piezas). Es decir, que la mitad de las monedas fueron producidas por poderes de época taifa que ejercieron su dominio sobre las islas. La pieza con la inscripción nafaqa fi sabil Allāh hallada en La Mola de Felanitx hace, por otro lado, verosímil la presencia de un ejemplar del mismo tipo en el lote del Museu de Menorca. De esta manera, de las escasas nafaqa-s conocidas, dos procederían de hallazgos baleares ${ }^{20}$. Finalmente, convendría reseñar la escasísima presencia de ejemplares de época califal en el conjunto atribuido a la colección Vives, acorde con los datos disponibles sobre los hallazgos de Ibiza y Menorca.

La inclusión de los fulūs del Museu de Menorca en el estudio de

17 Lavoi, H., Catalogue des monnaies musulmanes de la Bibliothèque Nationale, París, 1887 y 1891 (en adelante, Lavoix).

18 Tomo esta argumentación de Moll, B., "Contribució a l'estudi...".

19 Un estudio parcial de este conjunto se puede encontrar en Morgenstern, R., «Monedas árabes de bronce y plata del Museo de Menorca», Acta Numismàtica, 15 (1985), pp. 191-196.

20 M. Barceló identifica un total de ocho ejemplares. «Els fulūs de Țanğa...», p. 7. 
cuños (aunque sólo cuatro están en condiciones de ser contrastados) no hace sino confirmar la absoluta diversidad revelada por el primer ejercicio. Así pues, a la vista de estos primeros resultados, parece que, en las partidas de fulüs introducidas en las islas, se incorporaron piezas producidas en secuencias diferentes, en un lapso de tiempo, por ahora, imposible de determinar. La consideración, aunque con reservas, del lote de piezas del Museu de Menorca, permite insistir sobre esta cuestión. En efecto, la confirmación del origen menorquín del conjunto incrementaría, además, la diversidad tipológica de los fulūs. Aparte de los representados por los hallazgos catalogados, el lote incluye al menos cinco tipos más de piezas acuñadas, presumiblemente, en el Norte de Africa y al-Andalus ${ }^{21}$.

Mucho más claros, sin embargo, podrían ser los indicios proporcionados por las piezas omeyas de origen oriental, las emirales andalusíes y las procedentes del Norte de Africa anteriores al 290 H/902 d. C., fecha de la expedición de 'Ișām al-Jawlānī. Por lo que respecta a las monedas orientales, presentes de manera común en hallazgos peninsulares ${ }^{22}$, la única evidencia disponible de estas piezas se encuentra en el lote del Museu de Menorca. Aparte de dos fulūs sin nombre de ceca del $92 \mathrm{H}$, incluidos en este conjunto ${ }^{23}$, de posible origen oriental, hay que añadir la presencia de tres dirham-s omeyas [dos con el nombre de ceca Dimašq y las fechas 80 H y $92 \mathrm{H}$ (Walker, 3.53 y 368) y uno de Wāsit, probablemente acuñado en el $94 \mathrm{H}$ (Walker, 538)] y de un fals de Mișr, emitido sobre el $100 \mathrm{H}$ (Nützel, 2072) ${ }^{24}$. Esta evidencia de moneda califal omeya, escasa, es, no obstante, congruente con las proporciones contenidas en los ocultamientos de este período: Wāsit y Dimašq son,

21 Hay nueve ejemplares con la indicación de ceca الأندلس y trece más seguramente acuñados en el Norte de Africa o en al-Andalus: uno con la inscripción nafaqa y doce más también atribuibles a estas zonas (uno como Walker, 741-2) $(\mathrm{Pl} . \mathrm{XXV})=$ Lavoix, 1376-9; dos como Walker, $\mathrm{P} 116=$ Lavoix, 1342; tres como Walker, $740(\mathrm{Pl}$. XXV) = La-

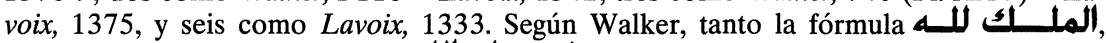
como la división de la leyenda محمد ر / سـول اللــ es a strong indication of coins of African or Spanish provenance. Walker, p. 226, nota P117. Sobre la atribución de las piezas nafaqa a la ceca de Țanğa, Barceló, M., "Els fulüs de Țanğa...», p. 8.

22 Véase, por ejemplo, los hallazgos de Azanuy (Huesca), Garraf (Barcelona), Granada o Yecla (Murcia). F. Mateu Llopis, "Hallazgos numismáticos musulmanes VIII", Al-Andalus, XVIII (1953) pp. 199-200, núms. 74 y 85; «Hallazgos numismáticos musulmanes VIII", Al-Andalus, XIX (1954) pp. 439-446; Morgenstern, R., "Hallazgo en la provincia de Granada de moneda de plata de los califas omeyas", Symposium Numismático de Barcelona, II, 1980, pp. 267-272.

23 Morgenstern, R., "Monedas árabes...», p. 192, núms. 22 y 39. Nützel, 2055-56.

${ }^{24}$ Morgenstern, R., "Monedas árabes...", núms. 77, 27, 28 y 29. 
precisamente, los nombres de ceca mayoritarios entre las piezas omeyas que circularon tanto en oriente como en el occidente islámico ${ }^{25}$.

En Ibiza fue hallada, en 1982, una pieza andalusí acuñada en el 176 H/792 d. C. a nombre de Hišām I. En el caso de Menorca, al ejemplar del año $167 \mathrm{H} / 783 \mathrm{~d}$. C. encontrado en Son Bou ${ }^{26}$, habría que añadir los cuatro recogidos en el trabajo de B. Moll, acuñados en los años 175 H/791 d. C., 211 H/826 d. C., 239 H/853 d. C y 257 H/870 d. C.

A estas piezas tanto orientales como andalusíes, hay que sumar los ejemplares procedentes de la parte occidental del Norte de Africa, uno de los cuales, una moneda de oro aglabí datable entre el 270-279 H/883-892 d. C., fue hallada en Menorca ${ }^{27}$. En el conjunto del Museu de Menorca hay, además, algunas piezas norteafricanas de los siglos IIIII H/VIII-IX d. C., entre las que destaca un fals rarísimo acuñado en Barqa (Walker, P125; Pl. XXVI), de cuyo tipo, sin embargo, existen numerosos ejemplares emitidos en Mosul entre los años 114-121 h/732-739 d. C. ${ }^{28}$. También forman parte de este lote un fals acuñado a nombre de Ibrāhīm I b. al-Aglab en al-'Abbāsīya (184-196 H; Lavoix, 829), cinco piezas de Idrīs II $(177-213 \mathrm{H}){ }^{29}$, y un dirham acuñado en Wazzaqūr a nombre de ${ }^{\mathrm{I}} \mathrm{I} a ̀ \mathrm{~b}$. Idrīs en el $244 \mathrm{o} 245$ H ${ }^{30}$.

La evidencia numismática considerada hasta ahora -con todas las reservas impuestas por los pocos ejemplares disponibles y por la incer-

25 Mientras que los nombres de ceca de al-Andalus e Ifrīqiya were always an exiguous, almost token, presence in the circulating stream. Es justamente la estructura de estos ocultamientos, común en las diferentes regiones sometidas al estado omeya de Damasco, una de las razones argüidas para proponer una oferta monetaria centralizada. El nombre de ceca, pues, no indicaría necesariamente el lugar de emisión de las piezas, sino que tendría some king of budgetary dimension. Barceló, M., "Coins from afar? New evidence on coin production and fiscal administrative practice in the Late Umayyad Caliphate", Problems of Medieval Coinage in the Iberian Area, 3, Santarém, (1988), pp. $107-$ 117 , p. 108.

26 Orfila, M.; Tuset, F., "Hallazgo de época islámica en Son Bou (Menorca)», Les Illes Orientals d'al-Andalus, Palma, 1987, pp. 185-190.

27 Moll, B., "Contribució a l'estudi..."

28 G. Rotter escribió sobre este tipo: The design of this fals is extremely rare. From the Umayyad period there is only other coin (from Barka in North Africa) with a similar geometrical design. G. Rotter, "The Umayyad fulūs of Mosul», Museum Notes, 19 (1974), pp. 165-198, p. 182. Ya en época 'abbāsí se acuñaron piezas, siguiendo el mismo modelo, en Rayy, Miles, G. C., The Numismatic History of Rayy, New York, 1938, p. 41.

29 Se trata de variantes inéditas, comentadas por Morgenstern en «Monedas árabes...", pp. 192-3.

30 Eustache, D., Corpus des dirhams idrisites et contemporains, Rabat, 1970-71, núms. 349-350. Morgenstern fecha esta pieza en el 224 h. "Monedas árabes...», p. 192. 
tidumbre sobre el origen del lote depositado en el Museu de Menorcapermite avanzar que, desde un momento indeterminado del siglo viII, pero seguramente no muy alejado del 711 , se producen entradas de piezas árabes en las Baleares. La diversidad de los cuños utilizados y la variedad tipológica de los fulūs iniciales, si se tiene en cuenta el conjunto del Museu de Menorca, sugieren que estas entradas se efectuaron siguiendo una cadencia, imposible, sin embargo, de precisar cronológicamente. En cualquier caso, resulta difícil pensar en una única entrada que incluyera la variedad de cuños y tipos indicada. La consideración de las piezas orientales, andalusíes y norteafricanas posteriores refuerza el descarte de esta posibilidad. Podría argüirse, no obstante, que, a pesar de tratarse de piezas acuñadas entre finales del siglo i $\mathrm{H}$ y el III $\mathrm{H}$, su introducción en las islas se produjera posteriormente; pongamos por caso, después de la conquista que siguió a la expedición de 'Ișām alJawlānī. La confirmación numismática de esta idea exigiría la existencia de depósitos, cerrados en época califal o posteriores, que incluyeran ejemplares de varios siglos antes. Esta circunstancia, al menos de momento, no se da en las islas, ni tampoco parece ser común en conjuntos peninsulares. De hecho, aunque en proporciones diferentes, la secuencia de los registros numismáticos de Ibiza y Menorca no difiere substancialmente de la establecida, por ejemplo, en el caso de los hallazgos provenientes de asentamientos de la zona de Crevillente (País Valenciano) ${ }^{31}$

Así pues, en principio, se puede proponer que entre el primer cuarto del siglo viI y principios del $\mathrm{x}$ se produjo un flujo de piezas de moneda hacia las Baleares. La escasa evidencia disponible sugiere, por otro lado, que estas entradas se sucedieron de manera intermitente, en proporciones modestas y que se circunscribieron a zonas precisas ${ }^{32}$. A pesar de las limitaciones de volumen, ritmo y distribución, el flujo parece que fue, sin embargo, persistente. Los cinco hallazgos de dirham-s

\footnotetext{
31 En el caso de los asentamientos de El Forat y Fontcalent, el registro cerámico concuerda cronológicamente con el numismático, probablemente desde el siglo viII y, con seguridad, desde el Ix. Gutiérrez, S., "La cerámica paleoandalusí del sureste peninsular (Tudmir): producción y distribución", La cerámica altomedieval en el Sur de al-Andalus, Granada, 1993, pp. 39-65, pp. 48-49. Por otro lado, en el yacimiento de El Frare, donde se ha encontrado el mayor número de piezas, destaca, significativamente, la cantidad de ejemplares de época taifa. Domènech, C.; Trelis, J., «Hallazgos numismáticos...».

$32 \mathrm{La}$ absoluta variedad de cuños proporciona información sobre los volúmenes globales de acuñación y sugiere la existencia de varias secuencias en la emisión; no es posible, sin embargo, deducir, de esta diversidad, una presencia voluminosa en las islas.
} 
emirales de Menorca, escalonados cronológicamente, ilustran claramente este proceso desde el último tercio del siglo II $\mathrm{H} /$ hasta, al menos, la mitad del III H. Es esta persistencia, precisamente, lo que convierte el registro numismático considerado, con todas las limitaciones apuntadas, en un conjunto evidencial compacto y, a la vez, en una cuestión por resolver.

La introducción en las islas de los fulūs de los siglos I y in H y el previsible mantenimiento de entradas de piezas en fases posteriores requirió la creación previa de un espacio monetario ${ }^{33}$. El contexto en el que se produjo la monetización -cuyos restos, hasta el momento, son bastante modestos- no pudo ser otro que el creado por los pactos, difíciles de datar con precisión, pero, en cualquier caso, anteriores a la expedición del 290 H/902 d. C. ${ }^{34}$.

La relación entre estos pactos, documentados en los casos de Mallorca y Menorca, y la evidencia numismática más temprana permite, en primer lugar, fechar el inicio de la intervención andalusí sobre las islas en un momento en el que los fulūs eran, todavía, especies monetarias en vigor; previsiblemente, pues, en unas fechas no demasiado alejadas de la emisión de los mismos ${ }^{35}$. Los sucesivos aportes posteriores, testificados por las piezas datables durante la segunda mitad del in $\mathrm{H}$ y mediados del III $\mathrm{H}$, marcan la vigencia de estos pactos a lo largo del período indicado.

Aparte de las consideraciones cronológicas, el registro numismático permite hacer observaciones sobre el contenido de los pactos. En primer lugar, resulta difícil explicar este registro prescindiendo de la exigencia de efectuar, en forma de moneda, al menos una parte de las

\footnotetext{
33 El espacio monetario sería aquél en el que, más allá de las especies físicas, se aceptan unidades de cuenta específicas -es decir, se establece un lenguaje monetario propio-y se reconocen las existencias de pago impuestas por un poder político. Aglietta, M.; Orlean, A., La violence de la monnaie, París, 1982, pp. 41 y 44. Entre otros artículos del mismo autor, véase Hennequin, G., «De la monnaie antique à la monaie musulmane», Annales E.S.C., 1975, pp. 890-899, y «Numismatique et monnaie pré-libérale: les limites d'un apport", $P A C T, 5$, Strasbourg, 1981, pp. 406-416.

${ }^{34}$ Sobre la cuestión de los pactos, sigo el reciente estudio de Barceló, M., «Els fulūs de Tanğa...".

35 Así, la fecha sugerida por el texto de Ibn al-Qūțiyya ( 95 h/715 d. C.) es verosímil Ta'rï iftitäh al-Andalus, ed. y trad. J. Ribera, p. 122 trad., p. 141 texto árabe. Barceló, M., "Els fulüs de Ṭanğa...", p. 15.
} 
cargas a que fueron sometidos los isleños ${ }^{36}$. Es precisamente el establecimiento de nexos fiscales lo que suscita la creación del espacio monetario, cuyos restos numismáticos, como parte de los pagos susceptibles de ser evaluados monetariamente, no son más que una señal tangible. No hay manera de explicar la reiterada -aunque magra- presencia de fulūs y dirham-s en las islas fuera de la lógica fiscal, a la que se supeditan, como alternativas explicativas, los pagos militares ${ }^{37} \mathrm{y}$ los intercambios ${ }^{38}$.

La constatación de este tipo de pago fiscal obliga, por otro lado, a plantear la cuestión del peso de esta forma de exacción respecto a otras cargas, sean éstas en forma de productos o de personas. Lo único claro es que el tercio exigido sobre amwāl y anfus (o darār) provocó en el $235 \mathrm{H} / 849$ d. C. la queja de las gentes de Mallorca y Menorca ( $a h l M a-$ yürqa wa-Minurqa) después de la renovación impuesta tras la intervención armada andalusí del $234 \mathrm{H}$. Precisamente la ruptura del acuerdo -entendida como la interrupción de los pagos debidos- fue, sin duda, lo que originó este ataque.

Parece ser, pues, que las condiciones recogidas en el pacto llegaron a suponer una carga difícil de soportar para los isleños, abatidos, según Ibn 'Id̄ārī, ante los pagos a los que estaban sujetos ${ }^{39}$. Es posible que la

36 Pagos que, presumiblemente, quedarían englobados como parte de los amwāl que, junto con anfus, eran los conceptos que fijaban las cargas tributarias que debían ser satisfechas. Sobre la interpretación de estos términos, incluidos en el resumen del pacto hęcho por Ibn Hayyān en el Muqtabis, véase Barceló, M., "Els fulūs de Țanğa...", pp. 9-10. Por otro lado, la primera organización fiscal musulmana en Egipto y Palestina contempló, desde el principio, la exigencia del pago de la ŷizya, entendida, antes de $c a$. 720 , como una tasa pagada con monedas. Simonsen, J. B., Studies in the Genesis and Early Development of the Caliphal Taxation System, Copenhagen, 1989, pp. 99 y 129131.

37 Por ejemplo, las acuñaciones efectuadas durante el período de la conquista han sido recientemente explicadas a partir de la necesidad de facilitar el reparto del botín entre las tropas. Chalmeta, P., Invasión e islamización, Madrid, 1994, p. 245.

38 Véase, por ejemplo, Reece, R., "Coins and Frontiers- or Supply and Demand", Aktes des XI Internationalen Limes Kongresses, Budapest, 1976, pp. 643-646; Hendy, M., "East and West: divergent models of coinage and its issue", Il secolo di Ferro, XXVIII Settimane di Studio del CISAM, Spoleto, 1991, pp. 637-674; Barceló, M., ""Rodes que giren dins el foc de l'infern" o per a què servia la moneda dels taifes?", Rubartayr, 0 (= Gaceta Numismática, 105-106), 1992, pp. 15-23. La relación entre registro numismático y moneda, sin embargo, no tiene por qué ser automática. Véase, por ejemplo, Blackburn, M. A. S., "Three Silver Coins in the Names of Valentinian III (425-55) and Anthemius (467-72) from Chatham Lines, Kent", Numismatic Chronicle, 148 (1988) pp. 169-174.

39 Ibn 'Id̄ārī, Kitāb al-bayān al-mugrib fì ajbār mulūk al-Andalus wa-l-Magrib, ed. E. Lévi-Provençal y G. S. Colin, 1948-51, vol. 2, p. 91; Barceló, M., «Els fulūs de Tanğa...», p. 15. 
entrega periódica de esclavos esté en el fondo del abatimiento y la resistencia ${ }^{40}$. Con todo, no es ésta la única exigencia capaz de afectar la estabilidad de las comunidades sujetas fiscalmente. El requerimiento de pagos hechos efectivos con monedas exige, como ya se ha explicado, la existencia previa de un espacio monetario, fijado por el monopolio sobre las unidades de tasación, el arbitrio sobre las especies aceptadas como pagos fiscales y el establecimiento de vías y de agentes transmisores y captadores de las monedas. La estrategia que rige todo este complejo montaje es el acceso a parte del trabajo campesino. Así, la presión tributaria obliga a generar excedentes que, una vez convertidos en moneda, permiten la satisfacción de las cargas monetarias ${ }^{4}$.

El registro numismático permite, de esta manera, plantear problemas que difícilmente pueden ser solucionados con el estricto estudio de las monedas. La cuestión que hay que resolver, pues, es el alcance de la presión fiscal monetaria ejercida; es decir, estimar la extensión territorial, la regularidad de las exacciones y la magnitud del trabajo capturado a través de la moneda. Por ahora, el registro numismático conocido tan sólo permite constatar el contenido monetario de una parte de las exigencias establecidas en los pactos. La monetización, sin embargo, parece que no fue impuesta más que en zonas limitadas y que no supuso la puesta en circulación de grandes cantidades de piezas.

Si la distribución restringida y el volumen modesto de la evidencia disponible se deben al predominio de otras formas de exacción o a la propia dispersión e insignificancia del poblamiento durante estos siglos ${ }^{42}$ (o lo que no es lo mismo, pero puede ser coincidente, a unos re-

40 Sobre la posibilidad de que los términos darāri-kum y anfusi-him hagan referencia a pagos efectuados en forma de personas, Barceló, M., "Els fulūs de Tanğa...", pp. 10 y 15 .

41 De aquí la importancia de incorporar - o de buscar- el registro numismático, especialmente el proporcionado por los hallazgos de piezas aisladas, perdidas involuntariamente, a las excavaciones y prospecciones arqueológicas. Razonablemente, las concentraciones significativas de ejemplares encontrados en zonas precisas permitiría la localización de los lugares -fundamentalmente, los mercados rurales- donde la moneda es adquirida a cambio de excedentes de trabajo. Será muy difícil, sin embargo, continuar confiando en las escasas, azarosas y normalmente mutiladas informaciones sobre los descubrimientos de piezas a la hora de programar líneas de investigación en este sentido.

42 En el caso de Ibiza, la evidencia arqueológica muestra un vacío absoluto de restos desde finales del siglo vII o comienzos del viI hasta bien entrado el siglo x. Ramon, J., "Ses Païsses de Cala d'Hort. Un establiment rural d'època antiga al Sud-Oest d'Eivissa", Quaderns d'Arqueologia Pitiüsa, 1, 1994. Por lo que respecta a Mallorca, la noticia de Ibn Hayyān sobre la expedición del 234 H/848-9 d. C. incluye una referencia a la gente 
ducidos niveles de exacción) son problemas cuya solución requiere estrategias que van más allá de la numismática. Los fulūs encontrados en Mallorca, Ibiza y Menorca sólo permiten, de momento, plantearlos ${ }^{43}$.

de los husūn. Ibn Jaldūn, de la misma manera, vuelve a utilizar el plural hușūn cuando relata la conquista de 'Ișām al-Jawlānī, en el 290 h/902 d. C. Sin embargo, de un texto de al-Zuhrī se desprende que la única resistencia significativa de los rūm de Mallorca se redujo a la presentada en el hiṣn Alarūn. Los indicios disponibles, al menos en los casos de Ibiza y Mallorca, sugieren, tal como indicara G. Rosselló hace años, que la población isleña de los siglos viII y Ix se vio reducida al mínimo. Rosselló, G., L'Islam a les Illes Baleares, Palma de Mallorca, 1968, p. 30; Barceló, M., "Comentaris a un text sobre Mayūrqa del geògraf al-Zuhrī (siglos vI-XII)", Sobre Mayūrqa, Palma, 1984, pp. 27-34, p. 29. Los relatos de Ibn Hayyān, al-Zuhrī e Ibn Jaldūn han sido recientemente comentados por Kirchner, H., en "Husün y alquerías campesinas en las Islas Orientales de al-Andalus», L'incastellamento: confronto fra società feudale e non feudale nel Mediterraneo Occidentale, Roma, 5-7 Maggio, 1994, en prensa.

43 Mientras este texto estaba en prensa, he tenido conocimiento de la existencia de un importante conjunto de monedas árabes halladas en la isla de Formentera. Se trata, como en los casos de Mallorca, Menorca e Ibiza, de piezas aisladas encontradas en superficie, no relacionadas, en principio, con estructuras arquitectónicas. El conjunto está compuesto por 23 monedas y dos ponderales. Un primer examen ha permitido distinguir, entre estos 23 ejemplares, 15 fulūs acuñados entre los siglos I y il $\mathrm{H}$. El tipo más representado es el correspondiente a Walker, 681, 684, presente también en Mallorca, Menorca e Ibiza. Hay un fals con una estrella de ocho puntas, seguramente acuñada en al-Andalus (Walker, 752), y un ejemplar, de origen andalusí o norteafricano, con la inscripción en el anverso bismilläh / al-mulk li-lläh (Walker, 741). La existencia de esta voluminosa evidencia numismática es congruente con la ya conocida en los casos de las otras islas y no hace sino reforzar las explicaciones presentadas en el texto. Sin embargo, tanto el desproporcionado volumen, en términos comparativos, de esta presencia de $f u$ lüs en Formentera como el hecho de que la mayor parte de los hallazgos haya tenido lugar en un radio apenas superior a un kilómetro obligan a plantear de manera específica el estudio de este peculiar conjunto monetario. 


\section{CATÁLOGO}

Núm. 1. Fals.
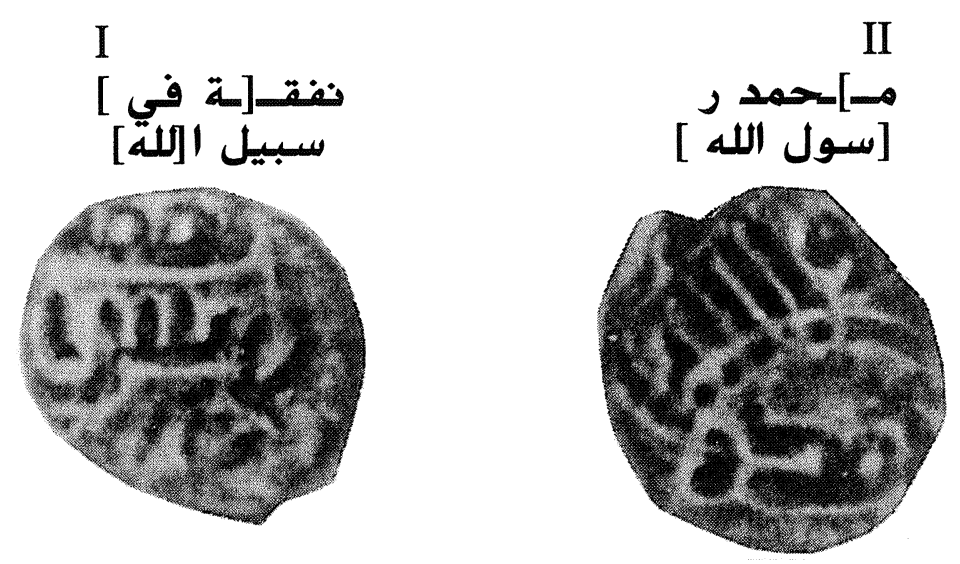

IIM:

4,20 gr.; 17 mm.; M. Barceló, «Els fulūs de Ṭanğa...», núm. 3.

Procedencia: La Mola de Felanitx (Mallorca).

Núm. 2. Fals.
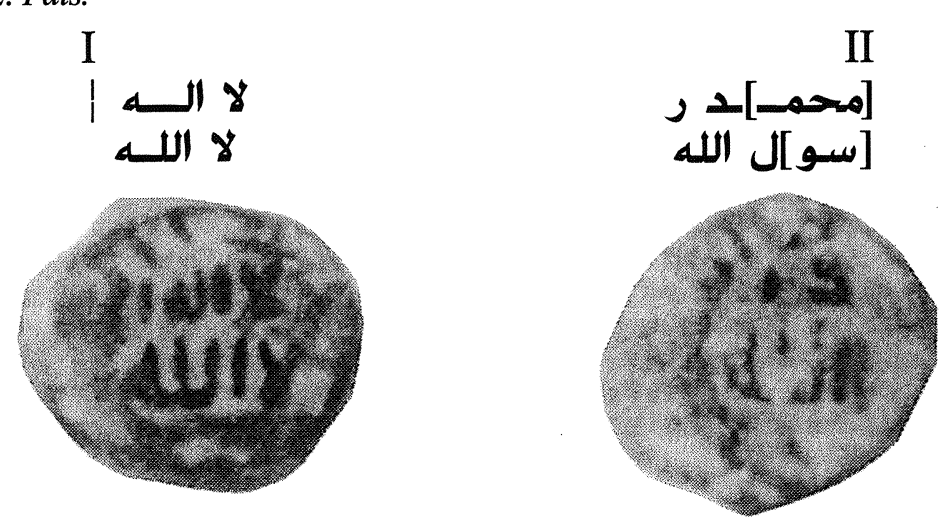

IIM:

3,05 gr.; 15 mm., 6 h.; Walker, 681, 684; Miles, 26.

Procedencia: La Mola de Felanitx (Mallorca). 
Núm. 3. Fals.

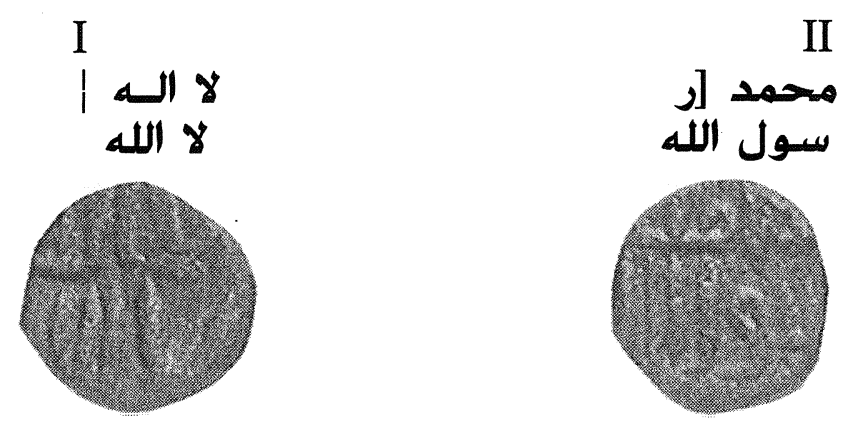

2,20 gr.; 12 mm.; 7 h.; Walker, 681, 684; Miles, 26.

Procedencia: Sa Coma (Ibiza). Museu Arqueològic d'Eivissa.

Núm. 4. Fals?
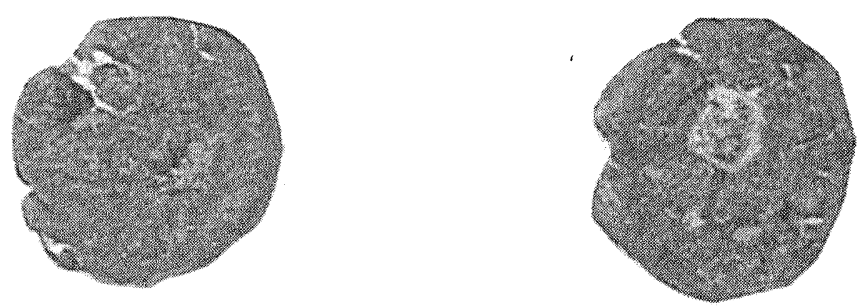

Pieza frustra. Procedencia: Sa Coma (Ibiza). Museu Arqueològic d'Eivissa. 
Núm. 5. Fals.
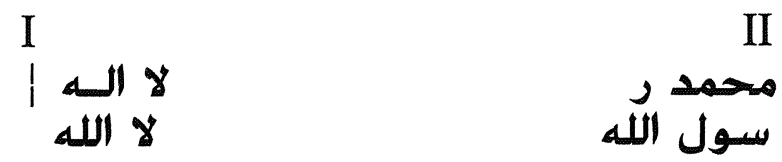

1,78 gr.; 12 mm.; 1 h.; B. Moll, "Contribució a l'estudi...”, núm. 1. Procedencia: Tot Lluc. Ciutadella (Menorca).

Núm. 6. Fals.
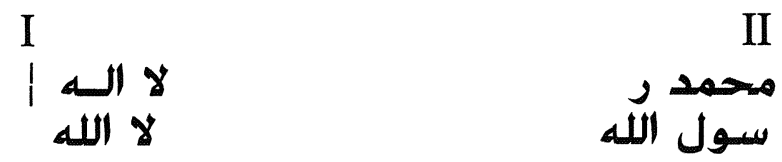

1,76 gr.; 12 mm.; 1 h.; B. Moll, «Contribució a l'estudi...», núm. 2.

Procedencia: Tot Lluc. Ciutadella (Menorca).

Agradezco al personal de los museos de Ibiza y de Menorca las facilidades dadas a la hora de consultar los fondos numismáticos depositados en estas instituciones.

\section{RESUMEN}

Este artículo trata de algunas piezas de cobre (fulūs) acuñadas entre del siglo i $\mathrm{H}$ y las primeras décadas del II $\mathrm{H}$ (VII-VIII d. C.) y halladas recientemente en Mallorca, Ibiza y Menorca. Esta evidencia numismática, junto con la proporcionada por un conjunto de piezas depositado en el Museu de Menorca, permite plantear cuestiones sobre la datación y el contenido de los pactos establecidos entre los andalusíes y los habitantes de las islas antes del año 902 d. C., fecha de la expedición de conquista de 'Ișām al-Jawlānī. 


\begin{abstract}
This article discusses some copper coins (fulūs) recently discovered in Mallorca, Ibiza and Menorca, minted between the end of the 1st and the early decades of the 2 nd centuries $\mathrm{H}$ ( 7 th-8th centuries AD). This new numismatic evidence, along with a collection of coins in the Menorca Museum, allows one to pose questions about the dates and contents of treaties established between the Andalusians and the inhabitants of the Islands before the conquest by 'Ișām alKhawlāni in $902 \mathrm{AD}$.
\end{abstract}

